\title{
Constrained Distributed Optimization Based on Population Dynamics
}

\author{
J. Barreiro-Gómez, N. Quijano, Senior Member, IEEE and C. Ocampo-Martinez, Senior Member, IEEE
}

\begin{abstract}
This paper proposes a novel methodology for solving constrained optimization problems in a distributed way, inspired by population dynamics and adding dynamics to the population masses. The proposed methodology divides the problem into smaller problems, whose feasible regions vary over time achieving an agreement to solve the global problem. The methodology also guarantees attraction to the feasible region and allows to have few changes in the decision-making design, when the network suffers the addition or removal of nodes. Simulation results are presented in order to illustrate several cases.
\end{abstract}

\section{INTRODUCTION}

An approach to design control systems is to express the desired performance of the plant as an optimization problem with multiple constraints, e.g., minimization of the error, minimization of the norm of states, minimization of the energy associated to control actions, all of those objectives subject to physical and/or operating constraints. When the system involves a large number of states, the design of optimization-based controllers becomes challenging, because of the lack of centralized information or because of other implications associated to information. The limitation regarding information availability demands the development of distributed optimization techniques that achieve an optimal point for the total system by using only local information.

There are many distributed optimization applications in engineering [2], and most of them are related to networked systems. These problems have been solved by using distributed optimization algorithms based on the Newton method [10], the subgradient method [11], and the consensus protocol [6]. On the other hand, game theory has become an important and powerful tool for solving optimization problems, e.g., the Nash equilibrium corresponds to the extreme of a potential function satisfying the Karush-KuhnTucker (KKT) first order condition [8]. Consequently, finding Nash equilibria based on local information allows to solve distributed optimization problems [1]. For instance, in [7], distributed optimization has been applied, using replicator dynamics based on local information. In [5] the design of utility functions for each agent in order to decouple constraints is presented, and the usage of penalty functions

J. Barreiro-Gomez and N. Quijano are with Departamento de Ingeniería Eléctrica y Electrónica, Universidad de los Andes, Carrera $1^{\mathrm{A}}$ No $18 \mathrm{~A}-10$ Bogotá, Colombia \{j.barreiro135, nquijano\}@uniandes.edu.co.

J. Barreiro-Gomez and C. Ocampo-Martinez are with the Automatic Control Department, Universitat Politècnica de Catalunya, Institut de Robòtica i Informàtica Industrial (CSIC-UPC), Llorens i Artigas, 4-6, 08028 Barcelona, Spain. \{jbarreiro, cocampo\}@iri.upc.edu.

J. Barreiro-Gómez is supported by COLCIENCIAS and by the Agència de Gestió d'Ajust Universitaris i de Recerca AGAUR. This work has been supported by the projects COLCIENCIAS 548/2012, and the EU project EFFINET (Ref. FP7-ICT-2011-8-31855). and barrier functions is discussed. The consideration of dynamics in the graph that describes information sharing among decision variables is paramount since some network systems in engineering may grow (e.g., drainage network systems, drinking water networks, distributed generation systems). These dynamics represent an addition or removal of elements to the network. Moreover, the connectivity of elements in the network could change over time (e.g., the system topology changes), which could affect availability of information. In [4], variations on the graph that determines the information sharing are studied, where the set of communication links varies with a certain probability.

The main contribution of this paper is a novel methodology to solve constrained optimization problems in a distributed way, inspired by population dynamics and adding dynamics to the population masses [8]. The global problem is represented by a society, where there is limitation of information sharing. The society is divided into several populations, where there is full information. Then, an optimization problem is solved at each population, whose feasible region varies dynamically, i.e., there is an interchange of masses among populations. The dynamic feasible regions vary until all populations agree to solve the global optimization problem. In addition to this, applications in control may involve disturbances that could lead the trajectories to leave the feasible region. The proposed method guarantees that the feasible region is attractive. Finally, the design of the decision-making distributed system allows to have a reduced number of modifications when the graph topology changes, i.e., when there are new nodes in the graph or there are nodes that disappear. Also, some redundant links can be identified, i.e., links in the graph that are not essential to solve the distributed problem.

The remainder of the paper is organized as follows. Section II presents preliminaries. Section III presents the population dynamics and the masses dynamics. Section IV presents the optimization problem forms that could be solved with the population dynamics and the masses dynamics, presenting also some illustrative examples and results. Finally, in Section $\mathrm{V}$ a discussion and the main conclusions are drawn.

\section{PRELIMINARIES}

Let $\mathcal{G}=(\mathcal{V}, \mathcal{E})$ be an undirected non-complete connected graph that exhibits the topology of a society, where $\mathcal{V}$ is the set of vertices that represents the set of strategies in a social game denoted by $S=\{1, \ldots, N\}$; and $\mathcal{E}=\{(i, j)$ : $i, j \in \mathcal{V}\}$ is the set of edges or links that determines possible interactions among strategies in the society. The graph $\mathcal{G}$ is divided into $M$ sub-complete graphs known as cliques [3], 
where each clique represents a population within the society. The set of populations is denoted by $\mathcal{P}=\{1, \ldots, M\}$ and the set of cliques is denoted by $\mathcal{C}=\left\{\mathcal{C}^{p}: p \in \mathcal{P}\right\}$, where $\mathcal{C}^{p}=\left(\mathcal{V}^{p}, \mathcal{E}^{p}\right)$. The set $\mathcal{V}^{p}$ represents the set of $N^{p}$ strategies in a population game denoted by $S^{p}=\left\{i: i \in \mathcal{V}^{p}\right\}$.

It is assumed that the cliques are already known, i.e., the number of cliques $M$, the set $\mathcal{V}^{p}$, and the set $\mathcal{E}^{p}$ for all $p \in \mathcal{P}$ are known. However, if it is desired to obtain the optimal set of cliques (i.e., the minimum amount of cliques $M$ such that $\cup_{p \in \mathcal{P}} \mathcal{V}^{p}=\mathcal{V}$ ), there are several methods. When cliques in a graph are identified, it is possible to find redundant links. A link $(i, j) \in \mathcal{E}$, is a redundant link if $(i, j) \notin \mathcal{E}^{p}$ for all $p \in \mathcal{P}$. The number of cliques containing a node $i \in \mathcal{V}$ is denoted by $G(i)=\sum_{p \in \mathcal{P}} g(i, p)$, where $g(i, p)=1$ if $i \in \mathcal{V}^{p}$ and $g(i, p)=0$ otherwise. Since $\mathcal{G}$ is a non-complete connected graph, all cliques must share at least one node with another clique. This node is known as an intersection node, where the set of intersection nodes in a population $p \in \mathcal{P}$ is denoted by $\mathcal{I}^{p}=\left\{i \in \mathcal{V}^{p}: G(i)>1\right\}$. The set of intersection nodes in $\mathcal{G}$ is $\mathcal{I}=\cup_{p \in \mathcal{P}} \mathcal{I}^{p}$.

Throughout the paper, all the populations $p$ are referred such that the node $i$ belongs to the set of nodes $\mathcal{V}^{p}$ (i.e., $p: i \in \mathcal{V}^{p}$ ), e.g., for a case with two populations whose sets of nodes are $\mathcal{V}^{1}=\{1,2\}$ and $\mathcal{V}^{2}=\{2,3\}$ respectively, then $\left\{p: 1 \in \mathcal{V}^{p}\right\}=\{1\}$, and $\left\{p: 2 \in \mathcal{V}^{p}\right\}=\{1,2\}$. The scalar $x_{i}$ is the amount of agents in the society selecting the strategy $i \in S$, and the scalar $x_{i}^{p}$ is the amount of agents selecting the strategy $i \in S^{p}$ in the population $p \in \mathcal{P}$. The distribution of agents throughout the available strategies is known as the social state and the population state denoted by $x \in \mathbb{R}^{N}$ and $x^{p} \in \mathbb{R}^{N^{p}}$, respectively. The set of social states is given by a simplex denoted by $\Delta$, which is a constant set, i.e., $\Delta=\left\{x \in \mathbb{R}_{+}^{N}: x^{\top} \mathbb{1}=m\right\}$, where $m$ is the constant mass of agents in the society and $\mathbb{1}$ is a column vector with unitary entries. The set of states of the population $p \in \mathcal{P}$ is given by a non-constant simplex denoted by $\Delta^{p}$, i.e., $\Delta^{p}=$ $\left\{x^{p} \in \mathbb{R}_{+}^{N^{p}}: x^{p \top} \mathbb{1}=m^{p}\right\}$, where $m^{p}$ corresponds to the mass of agents in the population $p$. There is a relationship between the social states and the population states, where $x_{i}^{p}=0$, if $i \notin \mathcal{V}^{p}$,

$$
x_{i}=\frac{1}{G(i)} \sum_{p \in \mathcal{P}} x_{i}^{p} .
$$

Let $F_{i}: \Delta \rightarrow \mathbb{R}$ be the fitness function for the proportion of agents playing strategy $i \in S$, and $F_{i}^{p}: \Delta^{p} \rightarrow \mathbb{R}$ is the fitness function for the proportion of agents playing strategy $i \in S^{p}$. The fitness for a strategy $i \in S$ is the same as the fitness for a strategy $j \in S^{p}$ if $i=j$. Consequently, for all $i \in S^{p}$ and $p: i \in \mathcal{V}^{p}$

$$
F_{i}(x)=F_{i}^{p}\left(x^{p}\right) \text {, if } x_{i}=x_{i}^{p} .
$$

The vector of fitness functions for the society is given by $F=\left\{F_{i}: i \in S\right\}, F \in \mathbb{R}^{N}$. The society average function denoted by $\bar{F}$ is $\bar{F}(x)=\left(x^{\top} F\right) / m$. The vector of fitness functions for a population $p \in \mathcal{P}$ is given by $F^{p}=\left\{F_{i}^{p}: i \in\right.$ $\left.S^{p}\right\}, F^{p} \in \mathbb{R}^{N^{p}}$, and the average function for a population $p \in \mathcal{P}$ is $\bar{F}^{p}\left(x^{p}\right)=\left(x^{p^{\top}} F^{p}\right) / m^{p}$. The relationship between the population masses and the social mass is given by

$$
m=\sum_{p \in \mathcal{P}} m^{p}-\sum_{i \in S}(G(i)-1) x_{i} .
$$

Remark 1: The relationship between the population masses and the social mass shown in (3) should be satisfied to guarantee that the simplex $\Delta$ is respected.

The framework of this paper is given by the assumptions stated below.

Assumption 1: $F(x)$ is a full potential game [8], i.e., there is a continuously differentiable function $f(x)$, known as the potential function, satisfying $\frac{\partial f(x)}{\partial x_{i}}=F_{i}(x)$ for all $i \in$ $S, x \in \Delta$.

Assumption 2: Fitness functions depend only on strategies that belong to the same clique.

Assumption 3: The population masses are strictly positive, i.e., $m^{p}>0$, for all $p \in \mathcal{P}$; and there is not extinction of proportion of agents playing a strategy, i.e., the scalar $x_{i}^{p}>0$ for all $p \in \mathcal{P}$, and $i \in S^{p}$.

Assumption 4: $F(x)$ is a stable game [8], i.e., the Jacobian matrix $D F(x)$ is negative semi-definite with respect to the tangent space defined as $T \Delta=\left\{z \in \mathbb{R}^{N}: \sum_{i \in S} z_{i}=0\right\}$, i.e., $z^{\top} D F(x) z \leq 0$, for all $z \in T \Delta$, and $x \in \Delta$. $\diamond$ The characteristics of the potential function $f(x)$ determine if the full potential game $F(x)$ is stable, i.e., if $f(x) \in C^{2}$ is concave, then the full potential game $F(x)$ is a stable game.

\section{POPULATION AND MASSES DYNAMICS}

The objective for the society is to converge to a Nash equilibrium denoted by $x^{*} \in \Delta$. In order to achieve this objective, there is going to be a game at each population $p \in \mathcal{P}$ converging to a Nash equilibrium denoted by $x^{p *} \in$ $\Delta^{p}$, and the intersection nodes $i \in \mathcal{I}$ are going to allow mass interchange between different populations.

\section{A. Population Dynamics}

A game is solved for each population with constraints given by masses $m^{p}$, which will vary dynamically. Dynamics associated to each population are shown in (4). There are $M$ dynamics of this type, one for each clique $\mathcal{C}^{p}$ for all $p \in \mathcal{P}$, i.e.,

$$
\begin{gathered}
\dot{x}_{i}^{p}=x_{i}^{p}\left(F_{i}^{p}-\bar{F}^{p}-\phi^{p}\right), \text { for all } i \in S^{p}, \\
\phi^{p}=\beta\left(\frac{1}{m^{p}} \sum_{j \in S^{p}} x_{j}^{p}-1\right),
\end{gathered}
$$

and $\beta$ is a convergence factor. Note that when $\phi^{p}=0$ (i.e., $\left.x^{p} \in \Delta^{p}\right)$, (4) is the replicator dynamics equation.

\section{B. Masses Dynamics}

On the other hand, the dynamics for population masses $m^{p}$ are given by

$$
\begin{aligned}
& \dot{m}_{i}^{p}=m_{i}^{p}\left(x_{i}-x_{i}^{p}-\psi_{i}\right), \text { for all } p: i \in \mathcal{V}^{p}, \\
& \psi_{i}=\beta\left(\frac{1}{\kappa_{i}+(G(i)-1) x_{i}} \sum_{q \in \mathcal{P}} \frac{m_{i}^{q}}{\left|\mathcal{I}^{q}\right|}-1\right) .
\end{aligned}
$$


Equation (6) describes the movements of agents among populations through intersection nodes, where $m_{i}^{q}=0$ if $q \notin \mathcal{V}^{q}$. The term $\beta$ is a convergence factor and forces the following equality at equilibrium

$$
\kappa_{i}+(G(i)-1) x_{i}^{*}=\sum_{q \in \mathcal{P}} \frac{m_{i}^{q *}}{\left|\mathcal{I}^{q}\right|},
$$

where $\kappa_{i} \in \mathbb{R}_{+}$is a distribution of the social mass $m$. Then, it should be satisfied that $\sum_{i \in \mathcal{I}} \kappa_{i}=m$. There is a relationship between $m_{i}^{p}$ for all $i \in \mathcal{I}^{p}$ and the population masses $m^{p}$ given by

$$
m^{p}=\frac{1}{\left|\mathcal{I}^{p}\right|} \sum_{i \in \mathcal{I}^{p}} m_{i}^{p}, \text { for all } p \in \mathcal{P} .
$$

For the masses dynamics at intersection nodes in (6), the vector of masses and the vector of states, that are associated to an intersection node $i \in \mathcal{I}$ are defined. The masses vector is $\mathrm{m}_{i}=\left\{m_{i}^{p}\right.$, for all $\left.p: i \in \mathcal{V}^{p}\right\}, \mathrm{m}_{i} \in \mathbb{R}^{G(i)}$, and the vector of population states is $\mathrm{x}_{i}=\left\{x_{i}^{p}\right.$, for all $\left.p: i \in \mathcal{V}^{p}\right\}$, $\mathrm{x}_{i} \in \mathbb{R}^{G(i)}$. Note that $\mathrm{m}_{i} \neq m_{i}$ and $\mathrm{x}_{i} \neq x_{i}$.

In order to illustrate the structure of the methodology and the interaction between the population dynamics and the masses dynamics, consider the social topology given by the graph $\mathcal{G}=(\mathcal{V}, \mathcal{E})$, where $\mathcal{V}=\{1,2,3,4,5\}$ and $\mathcal{E}=\{\{1,2\},\{1,3\},\{2,3\},\{3,4\},\{3,5\},\{4,5\}\}$. For this society, there are two populations and only one intersection node. Consequently, $\mathcal{I}^{1}=\mathcal{I}^{2}=\mathcal{I}=3$. The structure for this problem is shown in Figure 1.

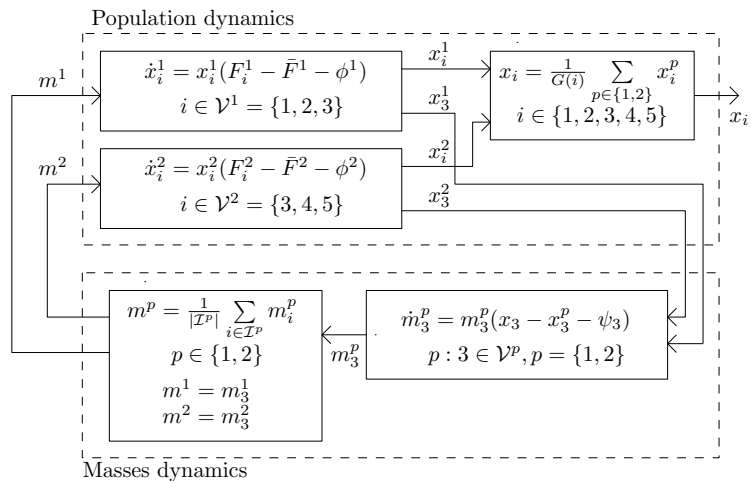

Fig. 1. The methodology structure with two population dynamics and one masses dynamics.

\section{Stability Analysis}

It is necessary to show that the solution of the distributed system with population dynamics (4) and masses dynamics (6) at intersection nodes, imply the solution of the social game (i.e. the global problem).

Proposition 1: If Assumption 3 is satisfied, the population dynamics are in equilibrium $x^{p *} \in \Delta^{p}$, for all $p \in \mathcal{P}$, and the masses dynamics are in equilibrium $\mathrm{m}_{i}^{*}$, for all $i \in \mathcal{I}$; then the society is in equilibrium $x^{*} \in \Delta$.

Proof: The equilibrium $x^{p^{*}} \in \Delta^{p}$ of the population dynamics (4), for all $p \in \mathcal{P}$, implies that $i) \phi^{p}\left(x^{p^{*}}\right)=0$, and, ii) $F_{i}^{p}\left(x^{p *}\right)=\bar{F}^{p}$, for all $i \in S^{p}$ and $p \in \mathcal{P}$. The equilibrium $\mathrm{m}_{i}{ }^{*}$ of the masses dynamics (6), for all $i \in \mathcal{I}$, implies that i) $\psi_{i}\left(x_{i}^{*}, \mathrm{~m}_{i}^{*}\right)=0$ since (8) is forced, and, ii) $x_{i}^{*}=x_{i}^{p *}$ for all $p: i \in \mathcal{V}^{p}$. Then, for all $i \in \mathcal{I}, x_{i}^{*}=x_{i}^{r *}=x_{i}^{k^{*}}$, for all $r: i \in \mathcal{V}^{r}$, and $k: i \in \mathcal{V}^{k}$. By (2) $F_{i}\left(x^{r *}\right)=F_{i}\left(x^{k^{*}}\right)=F_{i}\left(x^{*}\right)$. Moreover, $F_{i}\left(x^{r *}\right)=\bar{F}^{r}\left(x^{r *}\right)=F_{i}\left(x^{k^{*}}\right)=\bar{F}^{k}\left(x^{k^{*}}\right)$, for all $r, k \in \mathcal{P}$ and $i \in \mathcal{I}$. Consequently, all population average fitnesses are equal, then $F_{i}\left(x^{*}\right)=F_{j}\left(x^{*}\right)=\bar{F}\left(x^{*}\right)$ for all $i, j \in S$. Additionally, $\mathrm{m}_{i}^{*}$ for all $i \in \mathcal{I}$ satisfies (8). Then, $\sum_{i \in \mathcal{I}}\left(\kappa_{i}+(G(i)-1) x_{i}^{*}\right)=\sum_{i \in \mathcal{I}} \sum_{p \in \mathcal{P}} \frac{m_{i}^{p}}{\left|\mathcal{I}^{p}\right|}$. By (9), and using the fact that $m_{i}^{p}=0$, if $i \notin \mathcal{I}^{p}$, then

$\sum_{i \in \mathcal{I}} \kappa_{i}+\sum_{i \in \mathcal{I}}(G(i)-1) x_{i}^{*}=\sum_{p \in \mathcal{P}} m^{p}$. For a node $i \notin \mathcal{I}, G(i)=1$; and since $\sum_{i \in \mathcal{I}} \kappa_{i}=m, x^{p *} \in \Delta^{p}$, and owing that $x_{i}^{p}=0$, if $i \notin \mathcal{V}^{p}$

$$
m+\sum_{i \in S}(G(i)-1) x_{i}^{*}=\sum_{p \in \mathcal{P}} \sum_{i \in S^{p}} x_{i}^{p *}=\sum_{p \in \mathcal{P}} \sum_{i \in S} x_{i}^{p *},
$$

by Equation (1), $m+\sum_{i \in S}(G(i)-1) x_{i}^{*}=\sum_{i \in S} G(i) x_{i}^{*}$. Then $m=\sum_{i \in S} x_{i}^{*}$, and $x^{*} \in \Delta$ completes the proof.

Next, it is shown that the equilibrium points corresponding to the population dynamics and the masses dynamics are asymptotically stable.

Theorem 1: If $F(x)$ is a stable game, then there exists a $\beta$ such that the equilibrium point $x^{p^{*}} \in \Delta^{p}$ of the population dynamics (4), for all $p \in \mathcal{P}$, and the equilibrium point $\mathrm{m}_{i}{ }^{*}$ of the masses dynamics (6), for all $i \in \mathcal{I}$, are asymptotically stable.

Proof: Consider the convex radially unbounded Lyapunov function ${ }^{1}$

$$
\begin{array}{r}
V\left(x^{p}, \mathrm{~m}_{i}\right)=\sum_{p \in \mathcal{P}} \sum_{i \in S^{p}} x_{i}^{p}-x_{i}^{p *}\left(1+\ln \left(\frac{x_{i}^{p}}{x_{i}^{p^{*}}}\right)\right)+ \\
\sum_{i \in \mathcal{I}} \sum_{p: i \in \mathcal{V}^{p}} m_{i}^{p}-m_{i}^{p *}\left(1+\ln \left(\frac{m_{i}^{p}}{m_{i}^{p *}}\right)\right),
\end{array}
$$

where $V\left(x^{p *}, \mathrm{~m}_{i}{ }^{*}\right)=0$, and $V\left(x^{p}, \mathrm{~m}_{i}\right)>0$ if $x^{p} \neq x^{p *}$ or $\mathrm{m}_{i} \neq \mathrm{m}_{i}{ }^{*}$. Its derivative considering only one population and one intersection (to obtain the sum of negative elements),

$$
\dot{V}\left(x^{p}, \mathrm{~m}_{i}\right)=\sum_{i \in S^{p}}\left(1-\frac{x_{i}^{p *}}{x_{i}^{p}}\right) \dot{x}_{i}^{p}+\sum_{p: i \in \mathcal{V}^{p}}\left(1-\frac{m_{i}^{p *}}{m_{i}^{p}}\right) \dot{m}_{i}^{p} .
$$

Consider the column vector $\mathcal{I}_{i}=\left\{\frac{1}{\left|\mathcal{I}^{p}\right|}\right.$, for all $p: i \in$ $\left.\mathcal{V}^{p}\right\}, \mathcal{I}_{i} \in \mathbb{R}^{G(i)}$, and the change of variable $\tilde{\kappa}_{i}=\kappa_{i}+$ $(G(i)-1) x_{i}$, where $\tilde{\kappa}>0$. Then, We consider that $x^{p^{\top}} \mathbb{1}=$ $m^{p}+\epsilon$. The parameter $\epsilon$ could be either positive or negative depending on $x^{p}$. It is known that $x^{p *} \in \Delta^{p}$, then $x^{p * \top} \mathbb{1}=$ $m^{p}$. In the same way, it is possible that in a transitory event, $\mathrm{m}_{i}$ does not satisfy the condition in (8) Then, $\mathrm{m}_{i}^{\top} \mathcal{I}_{i}=\tilde{\kappa}_{i}+\gamma$, where $\gamma$ could be either positive or negative depending on condition in (8). Finally, since $\left|\mathcal{I}^{p}\right| \geq 1$, for all $p \in \mathcal{P}$, $\kappa_{i}+\gamma=\sum_{p \in \mathcal{P}} \frac{m_{i}^{p}}{\left|\mathcal{I}^{p}\right|} \leq \sum_{p \in \mathcal{P}} m_{i}^{p}=\kappa_{i}+\gamma+\theta$, where $\theta \geq 0$. Replacing $x^{p \top} \mathbb{1}, x^{p *^{\top}} \mathbb{1}, \mathrm{m}_{i}^{\top} \mathcal{I}_{i}$, and $\mathrm{m}_{i}^{\top} \mathbb{1}$,

$$
\begin{aligned}
\dot{V}\left(x^{p}, \mathrm{~m}_{i}\right)= & \alpha x^{p \top} F^{p}\left(x^{p}\right)-\beta \frac{\epsilon^{2}}{m^{p}}+\left(x^{p}-x^{p *}\right)^{\top} F^{p}\left(x^{p}\right)+ \\
& \frac{\gamma}{G(i)} \mathbf{x}_{i}^{\top} \mathbb{1}+\left(\mathrm{m}_{i}^{*}-\mathrm{m}_{i}\right)^{\top} \mathbf{x}_{i}-\beta \frac{\gamma^{2}}{\tilde{\kappa}_{i}},
\end{aligned}
$$

with $\alpha=\left(1-\frac{m^{p}+\epsilon}{m^{p}}\right)$. There are some cases to analyze:

\footnotetext{
${ }^{1}$ Details in this proof have been omitted due to the lack of space.
} 
i) $\epsilon=0$ and $\gamma=0$. Then $\dot{V}\left(x^{p}, \mathrm{~m}_{i}\right)=$ $\left(x^{p}-x^{p^{*}}\right)^{\top} F\left(x^{p}\right)+\left(\mathrm{m}_{i}^{*}-\mathrm{m}_{i}\right)^{\top} \mathrm{x}_{i}$. The first term is negative since $F\left(x^{p}\right)$ is a stable game. The second term is also negative since all fitness functions have the same decreasing tendency. ii) In all other cases, there exists a $\beta$ such that $\dot{V}\left(x^{p}, \mathrm{~m}_{i}\right) \leq 0$. For the case $\epsilon \neq 0$ and $\gamma \neq 0, \beta$ is

$$
\begin{aligned}
\beta \geq & \frac{m^{p} \tilde{\kappa}_{i}}{\tilde{\kappa}_{i} \epsilon^{2}+m^{p} \gamma^{2}}\left\{\left(1-\frac{m^{p}+\epsilon}{m^{p}}\right) x^{p \top} F^{p}\left(x^{p}\right)\right. \\
& \left.+\left(x^{p}-x^{p *}\right)^{\top} F^{p}\left(x^{p}\right)+\frac{\gamma}{G(i)} \mathbf{x}_{i}^{\top} \mathbb{1}+\left(\mathrm{m}_{i}^{*}-\mathrm{m}_{i}\right)^{\top} \mathbf{x}_{i}\right\} .
\end{aligned}
$$

Equality holds when $x^{p}=x^{p *}$ and $\mathrm{m}_{i}=\mathrm{m}_{i}^{*}$ (case i). Applying LaSalle's Invariance Principle, every solution starting in $x^{p}(0) \in \mathbb{R}_{+}^{N^{p}}$ and $\mathrm{m}_{i}(0) \in \mathbb{R}_{+}^{G(i)}$ approaches to $x^{p *}$ and $\mathrm{m}_{i}^{*}$ as $t \rightarrow \infty$. Note that when population states are near the feasible region (i.e., $\gamma \rightarrow 0, \epsilon \rightarrow 0$ ) the convergence factor is not longer required (i.e., $\beta$ can get any positive value or zero).

Remark 2: This proof also shows that the feasible region is attractive. i.e., if disturbances in the system make the trajectories to leave the feasible region, then the distributed system forces them to converge to a feasible solution.

\section{Changes in the graph}

Network systems in engineering are constantly growing. When this occurs, control systems have to be designed again in order to fit new conditions for the system. The method proposed in this paper allows to reduce the number of changes in design, when there are changes in the graph.

There are two cases to be considered: $a$ ) the addition of a clique to the graph; and $b$ ) the removal of a clique from the graph.

1) Addition of nodes to the graph: Consider the graph shown in Figure 2. For this graph, there are three populations (i.e., $\mathcal{C}=\left\{\mathcal{C}^{1}, \mathcal{C}^{2}, \mathcal{C}^{3}\right\}$, and $\mathcal{P}=\{1,2,3\}$ ) and two intersections (i.e., $\mathcal{I}=\{a, b\}$ ). Consequently, the decisionmaking system is composed by five dynamical systems.

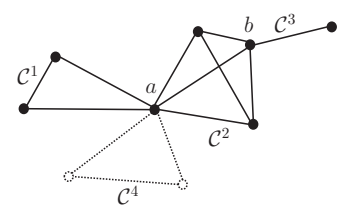

Fig. 2. Addition or removal of nodes in a graph. Dotted line represents the clique that is added or removed from the graph.

Now, suppose that there is a modification on the topology of the graph, by adding a new clique to the intersection node $a \in \mathcal{I}$. This change implies that masses dynamics designed for node $a$ should be modified, and that a new population dynamic system corresponding to clique $\mathcal{C}^{4}$ should be added to the decision-making system. The population dynamics designed for cliques $\mathcal{C}^{1}, \mathcal{C}^{2}$, and $\mathcal{C}^{3}$ (i.e., populations $p=$ $\{1,2,3\})$, and the masses dynamics for intersection node $b \in \mathcal{I}$ remain the same. Besides the fact that most of the dynamical systems do not need to be modified, when a clique is added, then the initial conditions for the variables associated to new nodes could get any value in $\mathbb{R}_{+}$without caring about the feasible region of the global problem. The decision-making system forces the trajectories to get in the feasible region. This property allows to design systems with connection an disconnection of cliques.

2) Removal of nodes to the graph: Now, suppose that the initial graph is shown in Figure 2, composed by four population (i.e., $\mathcal{C}=\left\{\mathcal{C}^{1}, \mathcal{C}^{2}, \mathcal{C}^{3}, \mathcal{C}^{4}\right\}$, and $\mathcal{P}=\{1,2,3,4\}$ ). Then, the decision-making system is compounded by six dynamical systems (i.e., four population dynamics and two masses dynamics). When population $p=4$ is removed from intersection node $a$, then this implies the elimination of one population dynamics system, and the modification of one mass dynamics system in node $a$. In this case, the population dynamics designed for cliques $\mathcal{C}^{1}, \mathcal{C}^{2}$ and $\mathcal{C}^{3}$ (i.e., populations $p=\{1,2,3\}$ ), and the masses dynamics for intersection node $b \in \mathcal{I}$ remain the same.

The removal of a clique could force the trajectories to leave the feasible region. However, the system adapts to changes satisfying the feasible region.

\section{OPTIMIZATION PROBLEMS WITH CONSTRAINTS}

One of the main features of full potential games is that their Nash equilibrium points correspond to extreme points of the potential function, i.e., Nash equilibria satisfy KKT first order conditions [8]. Additionally, if the potential function is concave, the games are stable and an optimization problem can be solved in a distributed way by using the population dynamics and the masses dynamics shown in Section III. Some optimization problem forms are set in this section, and illustrative examples are solved with the population dynamics and the masses dynamics described previously.

\section{A. Optimization problem with two constraints}

The general optimization problem related to population dynamics with full potential games is shown. This problem is a resource allocation problem, where $m$ is the total resource. The maximization problem is

$\max f(x)$, subject to $\sum_{i=1}^{N} x_{i}=m$, and $x \in \mathbb{R}_{+}^{N}$, where $f: \mathbb{R}_{+}^{N} \rightarrow \mathbb{R}$ and $m \in \mathbb{R}_{+}$. It is assumed that $f$ is continuously differentiable (i.e., $f \in C^{1}$ ) and concave. There is a full potential stable game given by $F(x)=\nabla f(x)$.

The first constraint in this optimization problem determines the set of social states $x \in \Delta$, and the second constraint is satisfied with population dynamics since the states are defined to be always positive. Constraints on information are given by a graph, which affects possible dependency among populations. An academical example of this type of problems is

$\max -x^{\top} A x+b x$

subject to $\sum_{i=1}^{13} x_{i}=522$, and $x \in \mathbb{R}_{+}^{13}$, where $A \in \mathbb{R}^{13 \times 13}$ is a sparse matrix with unitary diagonal and entries $a_{i, j}=1$ for $(i, j) \in\{(1,2),(6,7),(9,10),(11,12),(12,13)\}$, and $b=10\left[\begin{array}{llllllllll}5 & 4 & 1.2 & 3 & 6 & 7 & 8.5 & 5 & 2.1 & 3.5\end{array}\right.$ $2.62 .93 .2]$. For this optimization problem, there are limitations in information dependency given by the graph shown in Figure 4a). The results of convergence are shown 


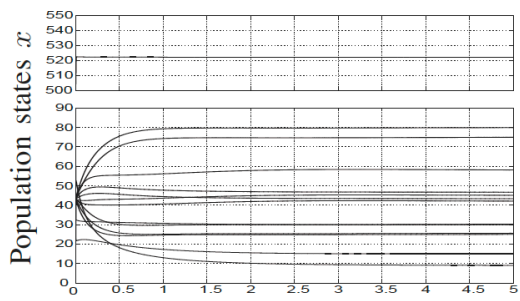

a)

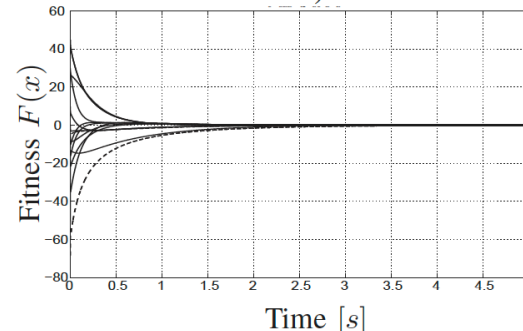

d)

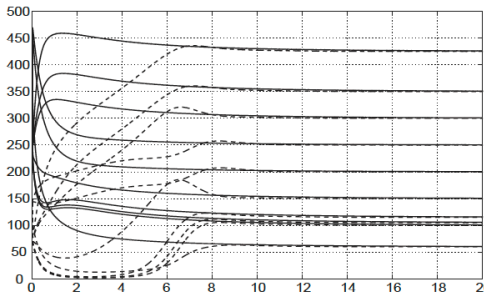

b)

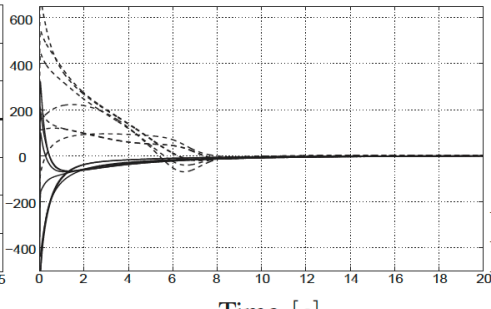

Time $[s]$

e)

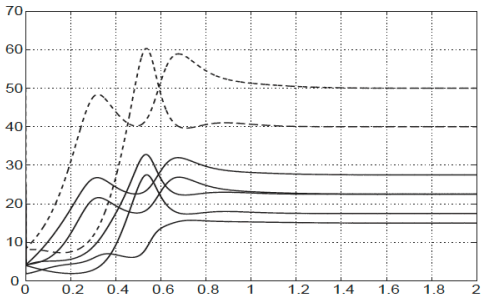

c)

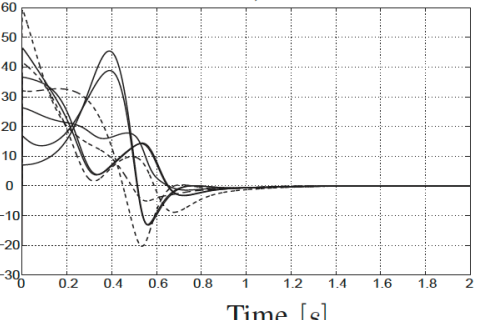

Time $[s]$

f)

Fig. 3. Population states evolution ( $a, b$ and $c$ ) and fitness functions evolution ( $d$, e and f) for different examples. Optimization problem with: two constraints (a and d), one constraint (b and e) and multiple constraints (c and f).

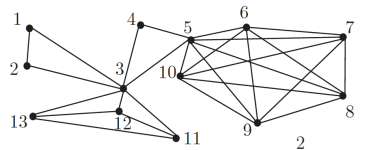

a)

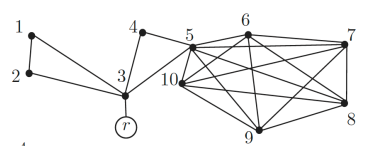

b) c)

Fig. 4. Social topology determining information availability at each node for examples.

in Figures 3a) and 3d). It can be seen that the constraint related to social mass is satisfied, even when population masses vary over time. Also, since dynamics are defined only for positive variables then the additional constraint related positiveness of variable is satisfied as well.

\section{B. Optimization problem with one constraint}

A less restrictive optimization problem is studied. This problem only demands the positiveness of variables. From a game theoretical perspective, it implies a variation of the social mass arbitrarily. The problem is

$\max f(x)$, subject to $x \in \mathbb{R}_{+}^{N}$,

where $f: \mathbb{R}_{+}^{N} \rightarrow \mathbb{R}$, and $f \in C^{1}$ is concave. Also, it is supposed that the optimal point of this problem is an interior point. Since it is not necessary to satisfy a determined social mass, then equality shown in (9) is not longer required. Consequently, the term $\psi_{i}$ in (6) is not necessary. Then, the masses dynamics are changed and rewritten as follows:

$$
\dot{m}_{i}^{p}=m_{i}^{p}\left(x_{i}-x_{i}^{p}\right) \text {, for all } p: i \in \mathcal{V}^{p} .
$$

With this modification, $\mathrm{m}_{i}^{*}$ still implies that $x_{i}=x_{i}^{p}$ for all $p: i \in \mathcal{V}^{p}$ and $i \in \mathcal{I}$. Also, the total system still converges to a Nash equilibrium since the population dynamics are the same. However, the social mass $m$ and the average fitness $\bar{F}(x)$ take arbitrary values at equilibrium.

The dynamical system can be forced to converge to a Nash equilibrium $x^{*}$ such that $\bar{F}\left(x^{*}\right)=\nabla f\left(x^{*}\right)$ converges to a desired value $F_{i}(r)$ for an $i \in \mathcal{I}$, where $r$ is a known value (e.g., a reference). Modifying the relationship between the states in (1) by adding the reference $r$, a new relationship is obtained, $x_{i}=\frac{1}{G(i)+1}\left(\sum_{p \in \mathcal{P}} x_{i}^{p}+r\right)$, where $x_{i}^{p}=0$, if $i \notin \mathcal{V}^{p}$. With this modification, and by (11), $x_{i}$ approaches to $r$. This makes $\bar{F}(x)$ to converge to the desired value $F_{i}(r)$, for only one $i \in \mathcal{I}$.

The solution for the optimization problem with one constraint is found by $F(x)=\nabla f(x)=0$, because $f(x)$ is concave and the fact that it is known that the maximum point is an interior point. Therefore, the desired value for average fitness is $F_{i}(r)=0$, and it is enough to find the correct value for reference $r$ for any intersection $i \in \mathcal{I}$.

Remark 3: In case that $r$ is not easily found for any $i \in \mathcal{I}$, it is possible to establish a known decreasing fictitious function denoted by $\tilde{F}_{N+1}\left(x_{N+1}\right)$, where $x_{N+1}$ is an auxiliary intersection node that is not part of the optimization problem. Reference $r$ is known, so that $\tilde{F}(r)$ is the desired value for average fitness.

The masses dynamics and the relationship between states have changed, then it is necessary to show stability for the new dynamical system.

Theorem 2: If $F(x)$ is a stable game, then there exists a $\beta$ such that the equilibrium $x^{p *}$ of the population dynamics (4) for all $p \in \mathcal{P}$, and the equilibrium $\mathrm{m}_{i}{ }^{*}$ of the masses dynamics (11) for all $i \in \mathcal{I}$ are asymptotically stable. $\diamond$ This proof can be made with the same Lyapunov function as in Theorem 1.

An academical example for this optimization problem form is

$\max -x^{\top} x+b x, \quad$ subject to $x \in \mathbb{R}_{+}^{10}$,

with $b=10^{2}\left[\begin{array}{llllllllll}5 & 4 & 1.2 & 3 & 6 & 7 & 8.5 & 2 & 2.1 & 2.3\end{array}\right]$. The graph for the social topology and possible information sharing for this optimization problem is shown in Figure 4b). It can be seen that the desired value of convergence is located at node three that is a decision variable that belongs to the 
optimization problem, i.e., it is known the argument of the fitness function three such that $F_{3}(r)=0$. Figure $3 \mathrm{~b}$ ) and $3 \mathrm{e}$ ) show convergence to the unique solution, from two different initial conditions. The results show convergence to the unique optimal point independently of initial conditions. Also, it is shown that fitness functions, which are the gradients of the concave potential function, converge to zero satisfying KKT conditions for an interior point.

\section{Optimization problem with multiple constraints}

Suppose that there is a strategic interaction with more than one constraint, e.g., it is required to converge to an equilibrium point. Also suppose that the total amount of certain groups of agent proportions are constant. This problem is $\max f(x)$, s. t. $A x=b$, and $x \in \mathbb{R}_{+}^{N}$, where $x \in \mathbb{R}_{+}^{N}$, $f: \mathbb{R}_{+}^{N} \rightarrow \mathbb{R}$, and $f \in C^{1}$ is concave. $A \in \mathbb{R}^{V \times N}$ since there are $V$ constraints and $N$ decision variables, and $b \in \mathbb{R}^{V}$. For this optimization problem, $\mu$ is the Lagrange multiplier vector. The Lagrange function $l: \mathbb{R}^{N} \times \mathbb{R}^{V} \rightarrow \mathbb{R}$ is $l(x, \mu)=$ $f(x)+\mu^{\top}(A x-b)$, and $\nabla_{x} l(x, \mu)=\nabla f(x)+A^{\top} \mu$, $-\nabla_{\mu} l(x, \mu)=-A x+b$. The Lagrange condition is used to find possible extreme points in the objective function, in which $\nabla_{x} l(x, \mu)=0, \nabla_{\mu} l(x, \mu)=0$. Consequently, fitness functions for each node are chosen as $F(x)=\nabla_{x} l(x, \mu)$, and $F(\mu)=\nabla_{\mu} l(x, \mu)$. This problem is solved by using references $r$ as it was explained in Section IV-B in order to force a convergence value for the fitness functions associated to the social states and the Lagrange multipliers. For both $F(x)$ and $F(\mu)$ a fictitious function can be set as explained in Remark 3. In order to use the population dynamics and the masses dynamics, it is necessary that the games are stable according to Assumption 3.

Lemma 1: If $f(x) \in C^{2}$ is concave, and constraints are of the form $A x=b$. Then the games $F(x)=\nabla_{x} l(x, \mu)$ and $F(\mu)=\nabla_{\mu} l(x, \mu)$ are stable.

Finally, optimization problems with constraints of the form $C x \leq d$ can be written as the form $A x=b$ by using slack variables [9]. An academical example for this optimization problem type is

$$
\begin{aligned}
& \max \left(25-x_{1}\right)^{2}+\left(20-x_{2}\right)^{2}+\left(15-x_{3}\right)^{2}+\left(10-x_{4}\right)^{2}+ \\
& \quad\left(5-x_{5}\right)^{2}, \\
& \text { subject to } x_{1}+x_{2}=50, x_{4}+x_{5}=40, \text { and } x \in \mathbb{R}_{+}^{5} .
\end{aligned}
$$

The graph corresponding to information limitation is shown in Figure 4c). There are nodes added to the graph corresponding to the Lagrange multipliers. For the Lagrange multipliers, there is an intersection node that is not part of the optimization problem. Then, this node contains a decreasing fictitious function $\tilde{F}$ with a known reference $r$ such that $\tilde{F}(r)=0$. The convergence of the population states to the solution can be seen in Figure 3c) and the convergence of fitness functions is shown in Figure 3f). The results show that there is a transitory event in which trajectories do not belong to the feasible region. However, the population dynamics and the masses dynamics force the trajectories to converge to the solution despite this fact. Additionally, the convergence time to the feasible region in this example is shorter than two seconds. Finally, Figure 3c) shows that both constraints are satisfied at same time (about $1.5 \mathrm{~s}$ ), what may be an advantage to verify constraints throughout the network.

\section{DisCUSSION AND CONCLUSIONS}

A methodology to solve different optimization problems with multiple constraints has been presented. The method is based on population dynamics, whose set of states is time varying. This variation represents a masses interchange among populations. The population dynamics and the masses dynamics are stable and the feasible region of the global problem is attractive, under the assumptions related to the objective function (i.e., potential function is concave). The distributed proposed decision-making system allows to design systems where there is connection and/or disconnection of cliques (which could be related to subsystems), without having to ensure initial conditions that satisfy constraints. A revision about whether or not the feasible region is satisfied to set initial conditions would require full information. The proposed methodology allows to initialize the distributed system with any value in $\mathbb{R}_{+}$. Additionally, it has been shown that changes in the network (e.g., the addition of a new clique or the variation of an existing clique) imply only local modifications in the decision-making system. Finally, there are some applications in which the feasible region should be satisfied all the time (e.g., physical constraints in a plant, constraints associated to an actuator, etc). In this case, the value of the convergence factor $\beta$ can be tuned to guarantee that the transitory event, where feasible region is not respected, is as fast as it is required. This ensures that when a decision is made, the feasible region is satisfied.

\section{REFERENCES}

[1] Gurdal Arslan and Jeff Shamma. Distributed convergence to Nash equilibria with local utility measurements. In In Proceedings of the Decision and Control, 2004. CDC. 43rd IEEE Conference on, volume 2, pages 1538-1543, 2004.

[2] Dimitri Bertsekas. Optimization for Machine Learning., chapter Incremental gradient, subgradient and proximal methods for convex optimization: a survey, pages 85-119. MIT Press, 2012.

[3] Immanuel M. Bomze, Marcello Pelillo, and Volker Stix. Approximating the maximum weight clique using replicator dynamics. IEEE Trans. Neural Netw. Learning Syst., 11(6):1228-1241, 2000.

[4] $\mathrm{Na} \mathrm{Li}$ and Jason Marden. Designing games for distributed optimization with a time varying communication graph. In In Proceedings of the Decision and Control, 2012 IEEE 51st Annual Conference on, pages 7764 - 7769, Dec 2012.

[5] Na Li and Jason Marden. Decoupling coupled constraints through utility design. Transactions on Automatic Control, pages 1-6, 2014.

[6] Giuseppe Notarstefano and Francesco Bullo. Distributed abstract optimization via constraints consensus: Theory and applications. IEEE Transaction Automatic Control, 56(10):2247-2261, 2011.

[7] A. Pantoja and N. Quijano. A population dynamics approach for the dispatch of distributed generators. IEEE Transactions on Industrial Electronics, 58(10):4559-4567, 2011.

[8] William H. Sandholm. Population games and evolutionary dynamics. Economic learning and social evolution. Cambridge. MIT Press, 2010.

[9] Jing Wang and Nicola Elia. A control perspective for centralized and distributed convex optimization. In In Proceedings of Decision and Control and European Control Conference (CDC-ECC), 2011 50th IEEE Conference on, pages 3800-3805. IEEE, 2011.

[10] Ermin Wei, Asuman E. Ozdaglar, and Ali Jadbabaie. A distributed Newton method for network utility maximization-I: Algorithm. IEEE Transaction Automatic Control, 58(9):2162-2175, 2013.

[11] Minghui Zhu and Sonia Martínez. On distributed convex optimization under inequality and equality constraints. IEEE Transaction Automatic Control, 57(1):151-164, 2012. 